\title{
Observation of the Vacuum Rabi Spectrum for One Trapped Atom
}

\author{
A. Boca, R. Miller, K. M. Birnbaum, A. D. Boozer, J. McKeever, and H. J. Kimble \\ Norman Bridge Laboratory of Physics 12-33, California Institute of Technology, Pasadena, CA 91125, USA
}

(Received 19 October 2004; published 3 December 2004)

\begin{abstract}
The transmission spectrum for one atom strongly coupled to the field of a high finesse optical resonator is observed to exhibit a clearly resolved vacuum Rabi splitting characteristic of the normal modes in the eigenvalue spectrum of the atom-cavity system. A new Raman scheme for cooling atomic motion along the cavity axis enables a complete spectrum to be recorded for an individual atom trapped within the cavity mode, in contrast to all previous measurements in cavity QED that have required averaging over $10^{3}-10^{5}$ atoms.
\end{abstract}

DOI: $10.1103 /$ PhysRevLett.93.233603

PACS numbers: 42.50.Pq, 03.67.-a, 32.80.Pj

A cornerstone of optical physics is the interaction of a single atom with the electromagnetic field of a high quality resonator. Of particular importance is the regime of strong coupling, for which the frequency scale $g$ associated with reversible evolution for the atom-cavity system exceeds the rates $(\gamma, \kappa)$ for irreversible decay of atom and cavity field, respectively [1]. In the domain of strong coupling, a photon emitted by the atom into the cavity mode is likely to be repeatedly absorbed and reemitted at the single-quantum Rabi frequency $2 g$ before being irreversibly lost into the environment. This oscillatory exchange of excitation between atom and cavity field results from a normal-mode splitting in the eigenvalue spectrum of the atom-cavity system [2-4], and has been dubbed the vacuum Rabi splitting [3].

Strong coupling in cavity QED as evidenced by the vacuum Rabi splitting provides enabling capabilities for quantum information science, including for the implementation of scalable quantum computation $[5,6]$, for the realization of distributed quantum networks $[7,8]$, and more generally, for the study of open quantum systems [9]. Against this backdrop, experiments in cavity QED have made great strides over the past two decades to achieve strong coupling [10]. The vacuum Rabi splitting for single intracavity atoms has been observed with atomic beams in both the optical [11-13] and microwave regimes [14]. The combination of laser cooled atoms and large coherent coupling has enabled the vacuum Rabi spectrum to be obtained from transit signals produced by single atoms [15]. A significant advance has been the trapping of individual atoms in a regime of strong coupling $[16,17]$, with the vacuum Rabi splitting first evidenced for single trapped atoms in Ref. [16] and the entire transmission spectra recorded in Ref. [18].

Without exception these prior single atom experiments related to the vacuum Rabi splitting in cavity QED [1118] have required averaging over trials with many atoms to obtain quantitative spectral information, even if individual trials involved only single atoms (e.g., $10^{5}$ atoms were required to obtain a spectrum in Ref. [14] and $>10^{3}$ atoms were needed in Ref. [18]). By contrast, the imple- mentation of complex algorithms in quantum information science requires the capability for repeated manipulation and measurement of an individual quantum system, as has been spectacularly demonstrated with trapped ions $[19,20]$ and recently with Cooper pair boxes [21].

With this goal in mind, in this Letter we report measurements of the spectral response of single atoms that are trapped and strongly coupled to the field of a high finesse optical resonator. By alternating intervals of probe measurement and of atomic cooling, we record a complete probe spectrum for one-and-the-same atom. The vacuum Rabi splitting is thereby measured in a quantitative fashion for each atom by way of a protocol that represents a first step towards more complex tasks in quantum infor-

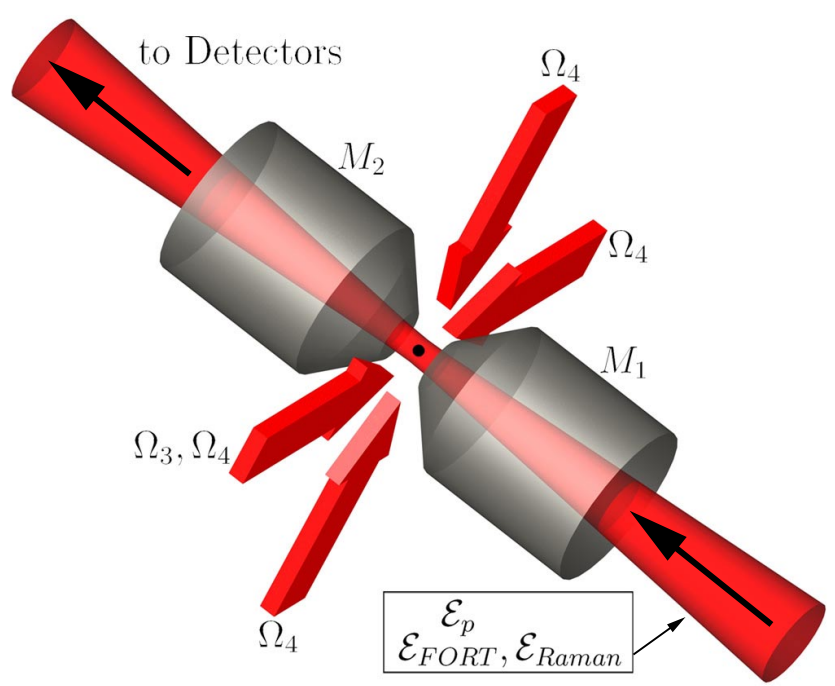

FIG. 1 (color online). A single atom is trapped inside an optical cavity in the regime of strong coupling by way of an intracavity FORT driven by the field $\mathcal{E}_{\mathrm{FORT}}$. The transmission spectrum $T_{1}\left(\omega_{p}\right)$ for the atom-cavity system is obtained by varying the frequency $\omega_{p}$ of the probe beam $\mathcal{E}_{p}$ and recording the output with single-photon detectors. Cooling of the radial atomic motion is accomplished with the transverse fields $\Omega_{4}$, while axial cooling results from Raman transitions driven by the fields $\mathcal{E}_{\mathrm{FORT}}, \mathcal{E}_{\text {Raman }}$. An additional transverse field $\Omega_{3}$ acts as a repumper during probe intervals. 
mation science. An essential component of our protocol is a new Raman scheme for cooling atomic motion along the cavity axis that leads to inferred atomic localization $\Delta z_{\text {axial }} \simeq 33 \mathrm{~nm}, \Delta \rho_{\text {transverse }} \simeq 5.5 \mu \mathrm{m}$.

A simple schematic of our experiment is given in Fig. 1 [22]. After release from a magneto-optical trap (MOT) located several $\mathrm{mm}$ above the Fabry-Perot cavity formed by mirrors $\left(M_{1}, M_{2}\right)$, single Cesium atoms are cooled and loaded into an intracavity far-off-resonance trap (FORT) and are thereby strongly coupled to a single mode of the cavity. Our experiment employs the $6 S_{1 / 2}, F=4 \rightarrow$ $6 P_{3 / 2}, F^{\prime}=5^{\prime}$ transition of the $D 2$ line in Cs at $\lambda_{A}=$ $852.4 \mathrm{~nm}$, for which the maximum single-photon Rabi frequency $2 g_{0} / 2 \pi=68 \mathrm{MHz}$ for $\left(F=4, m_{F}= \pm 4\right) \rightarrow$ $\left(F^{\prime}=5^{\prime}, m_{F}^{\prime}= \pm 5\right)$. The transverse decay rate for the $6 P_{3 / 2}$ atomic states is $\gamma / 2 \pi=2.6 \mathrm{MHz}$, while the cavity field decays at rate $\kappa / 2 \pi=4.1 \mathrm{MHz}$. Our system is in the strong coupling regime of cavity QED $g_{0} \gg(\gamma, \kappa)[1]$.

The intracavity FORT is driven by a linearly polarized input field $\mathcal{E}_{\mathrm{FORT}}$ at $\lambda_{F}=935.6 \mathrm{~nm}$ [23], resulting in nearly equal ac-Stark shifts for all Zeeman states in the $6 S_{1 / 2}, F=3,4$ manifold [24]. At an antinode of the field, the peak value of the trapping potential for these states is $U_{0} / h=-39 \mathrm{MHz}$ for all our measurements. Zeeman states of the $6 P_{3 / 2}, F^{\prime}=5^{\prime}$ manifold likewise experience a trapping potential, albeit with a weak dependence on $m_{F}^{\prime}$ [17]. The cavity length is independently stabilized to length $l_{0}=42.2 \mu \mathrm{m}$ such that a $\mathrm{TEM}_{00}$ mode at $\lambda_{C_{1}}$ is resonant with the free-space atomic transition at $\lambda_{A}$ and another $\mathrm{TEM}_{00}$ mode at $\lambda_{C_{2}}$ is resonant at $\lambda_{F}$ [25].

As illustrated in Fig. 1, we record the transmission spectrum $T_{1}\left(\omega_{p}\right)$ for a weak external probe $\mathcal{E}_{p}$ of variable frequency $\omega_{p}$ incident upon the cavity containing one strongly coupled atom [26]. Our protocol consists of an alternating sequence of probe and cooling intervals. The probe beam is linearly polarized [27] and is matched to the $\mathrm{TEM}_{00}$ mode around $\lambda_{C_{1}}$. $\mathcal{E}_{p}$ illuminates the cavity for $\Delta t_{\text {probe }}=100 \mu \mathrm{s}$, and the transmitted light is detected by photon counting [28]. During this interval a repumping beam $\Omega_{3}$, transverse to the cavity axis and resonant with $6 S_{1 / 2}, F=3 \rightarrow 6 P_{3 / 2}, F^{\prime}=4^{\prime}$, also illuminates the atom. In successive probe intervals, the frequency $\omega_{p}$ is linearly swept from below to above the common atom-cavity resonance at $\omega_{A} \simeq \omega_{C_{1}}$. The frequency sweep for the probe is repeated eight times in $\Delta t_{\text {tot }}=1.2 \mathrm{~s}$, and then a new loading cycle is initiated.

Following each probe interval, we apply light to cool both the radial and axial motion for $\Delta t_{\text {cool }}=2.9 \mathrm{~ms}$. Radial cooling is achieved by the $\Omega_{4}$ beams consisting of pairs of counter-propagating fields in a $\sigma_{ \pm}$configuration perpendicular to the cavity axis, as shown in Fig. 1. The $\Omega_{4}$ beams are detuned $\Delta_{4} \simeq 10 \mathrm{MHz}$ to the blue of the $4 \rightarrow 4^{\prime}$ transition to provide blue Sisyphus cooling [29] for motion transverse to the cavity axis.

To cool the axial motion for single trapped atoms, we have developed a new scheme that employs $\mathcal{E}_{\mathrm{FORT}}$ and an auxiliary field $\mathcal{E}_{\text {Raman }}$ that is frequency offset by $\Delta_{\text {Raman }}=$ $\Delta_{H F}+\delta$ and phase locked to $\mathcal{E}_{\mathrm{FORT}}$. Here, $\Delta_{H F}=$ $9.192632 \mathrm{GHz}$ is the hyperfine splitting between $6 S_{1 / 2}, F=3,4$. $\mathcal{E}_{\mathrm{FORT}}, \mathcal{E}_{\text {Raman }}$ drive Raman transitions between the $F=3$, 4 levels with effective Rabi frequency $\Omega_{E} \sim 200 \mathrm{kHz}$. By tuning $\delta$ near the $\Delta n=-2$ motional sideband (i.e., $-2 \nu_{0} \sim \delta=-1.0 \mathrm{MHz}$, where $\nu_{0}$ is the axial vibrational frequency at an antinode of the FORT), we implement sideband cooling via the $F=3 \rightarrow 4$ transition, with repumping provided by the $\Omega_{4}$ beams. The Raman process also acts as a repumper for population pumped to the $F=3$ level by the $\Omega_{4}$ beams. Each cooling interval is initiated by turning on the fields $\Omega_{4}, \mathcal{E}_{\text {Raman }}$ during $\Delta t_{\text {cool }}$ and is terminated by gating these fields off before the next probe interval $\Delta t_{\text {probe }}$.

Figure 2 displays normalized transmission spectra $T_{1}$ [26] for individual atoms acquired by alternating probe and cooling intervals. Clearly evident in each trace is a two-peaked structure that represents the vacuum Rabi splitting observed on an atom-by-atom basis. Also shown is the predicted transmission spectrum obtained from the steady-state solution to the master equation for one atom strongly coupled to the cavity, as discussed below. The quantitative correspondence between theory and experiment is evidently quite reasonable for each atom. Note that $m_{F}$-dependent Stark shifts for $F^{\prime}=5^{\prime}$ in conjunction with optical pumping caused by $\mathcal{E}_{p}$ lead to the asymmetry of the peaks in Fig. 2 via an effective population-dependent shift of the atomic resonance frequency [30].

To obtain the data in Fig. $2, N_{\text {load }}=61$ atoms were loaded into the FORT in 500 attempts, with the probabil-

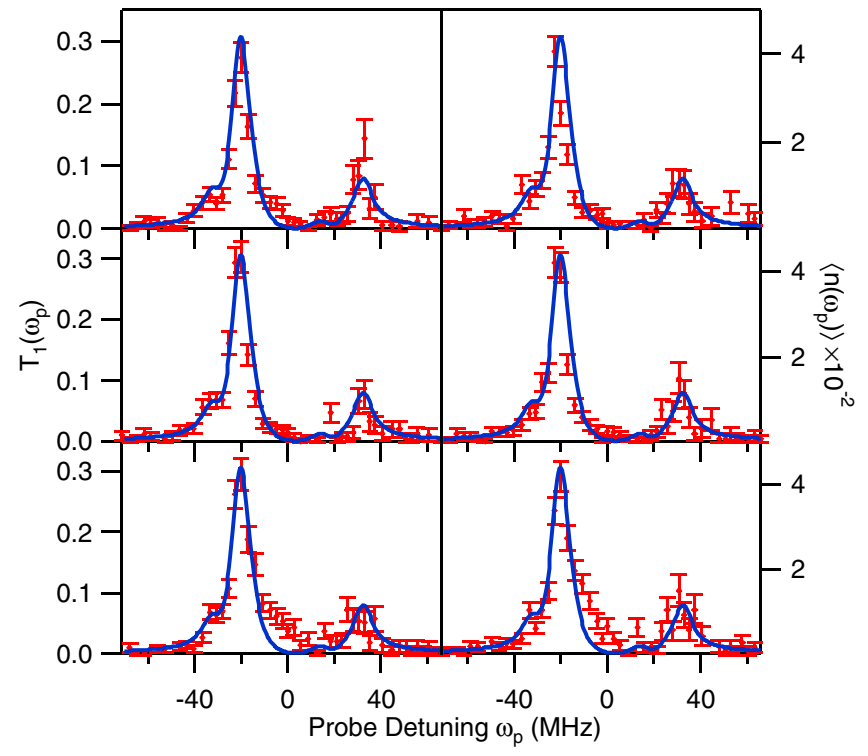

FIG. 2 (color online). Transmission spectrum $T_{1}\left(\omega_{p}\right)$ for six randomly drawn atoms [26]. In each case, $T_{1}\left(\omega_{p}\right)$ is acquired for one-and-the-same atom, with the two peaks of the vacuum Rabi spectum clearly evident. The error bars reflect the statistical uncertainties in the number of photocounts. The full curve is from the steady-state solution to the master equation. 
ity that a given successful attempt involved two or more atoms estimated to be $P_{\text {load }}(N \geq 2) \lesssim 0.06$. Of the $N_{\text {load }}$ atoms, $N_{\text {survive }}=28$ atoms remained trapped for the entire duration $\Delta t_{\text {tot }}$. The six spectra shown in Fig. 2 were selected by a random drawing from this set of $N_{\text {survive }}$ atoms. Our sole selection criterion for presence of an atom makes no consideration of the spectral structure of $T_{1}\left(\omega_{p}\right)$ except that there should be large absorption on line center, $T_{1}\left(\omega_{p}=\omega_{C_{1}}\right) \leq T_{\text {thresh }} \approx 0.2$ [26,31]. Note that an atom trapped in the FORT in the absence of the cooling and probing light has lifetime $\tau_{0} \simeq 3 \mathrm{~s}$, which leads to a survival probability $p\left(\Delta t_{\text {tot }}\right) \simeq 0.7$.

In Fig. 3 we collect the results for $T_{1}\left(\omega_{p}\right)$ for all $N_{\text {survive }}=28$ atoms, and display the average transmission spectrum $\bar{T}_{1}\left(\omega_{p}\right)$, as well as a scatter plot from the individual spectra. This comparison demonstrates that the vacuum Rabi spectrum observed for any particular atom represents with reasonable fidelity the spectrum that would be obtained from averaging over many atoms, albeit with fluctuations due to Poisson counting and optical pumping effects over the finite duration of the probe. The total acquisition time associated with the probe beam for the spectrum of any one atom is only $40 \mathrm{~ms}$.

We have also acquired transmission spectra $T_{1}\left(\omega_{p}\right)$ for operating conditions other than those in Figs. 2 and 3 , including intensities $\left|\mathcal{E}_{p}\right|^{2}$ varied by factors of 2 , $\frac{1}{2}$, and $\frac{1}{4}$, and atom-cavity detunings $\Delta_{A C}=\omega_{A}-\omega_{C_{1}}=$ $\pm 13 \mathrm{MHz}$. We will describe these results elsewhere.

The full curves in Figs. 2 and 3 are obtained from the steady-state solution of the master equation including all transitions $\left(F=4, m_{F}\right) \leftrightarrow\left(F^{\prime}=5^{\prime}, m_{F}^{\prime}\right)$ with their respective coupling coefficients $g_{0}^{\left(m_{F}, m_{F}^{\prime}\right)}$, as well as the two nearly degenerate modes of our cavity [23,27]. For

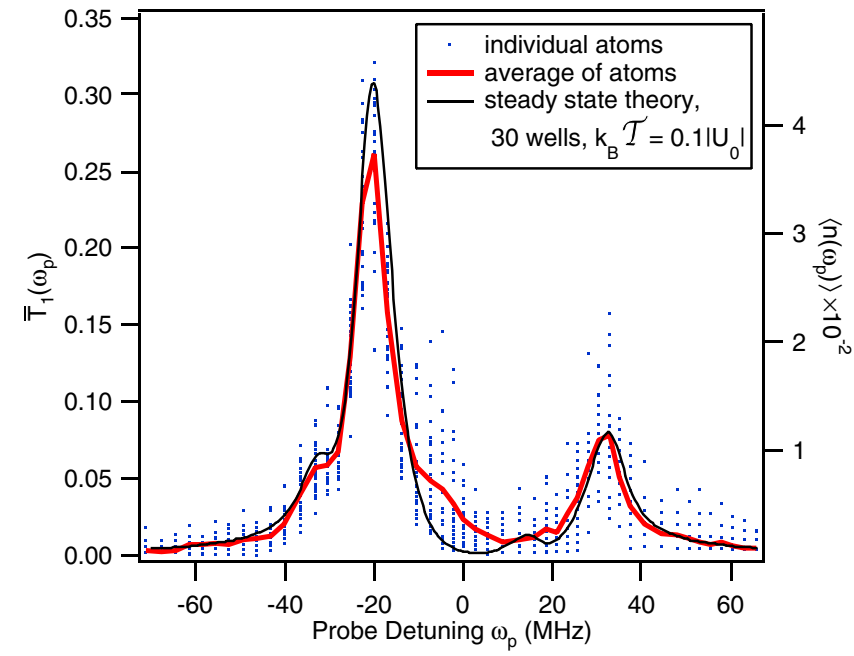

FIG. 3 (color online). Transmission spectrum $\bar{T}_{1}\left(\omega_{p}\right)$ (thick trace) resulting from averaging all 28 individual spectra $T_{1}\left(\omega_{p}\right)$ (dots) [26]. The thin trace is from the steady-state solution to the master equation, and is identical to that in Fig. 2. The only free parameters in the theory are the temperature and the range of FORT antinodes; the vertical scale is absolute. the comparison of theory and experiment, the parameters $\left(g_{0}^{\left(m_{F}, m_{F}^{\prime}\right)}, \gamma, \kappa, \Delta_{A C}, \omega_{p}-\omega_{A},\left|\mathcal{E}_{p}\right|^{2}, U_{0}\right)$ are known in absolute terms without adjustment. However, we have no $a$ priori knowledge of the particular FORT well into which the atom is loaded along the cavity standing wave, nor of the energy of the atom. The FORT shift and coherent coupling rate are both functions of atomic position $\mathbf{r}$, with $U(\mathbf{r})=U_{0} \sin ^{2}\left(k_{C_{2}} z\right) \exp \left(-2 \rho^{2} / w_{C_{2}}^{2}\right) \quad$ and $g^{\left(m_{F}, m_{F}^{\prime}\right)}(\mathbf{r})=$ $g_{0}^{\left(m_{F}, m_{F}^{\prime}\right)} \psi(\mathbf{r})$, where $g_{0}^{\left(m_{F}, m_{F}^{\prime}\right)}=g_{0} G_{m_{F}, m_{F}^{\prime}}$ with $G_{i, f}$ related to the Clebsch-Gordan coefficient for the particular $m_{F} \leftrightarrow m_{F}^{\prime}$ transition. $\psi(\mathbf{r})=\cos \left(k_{C_{1}} z\right) \exp \left(-\rho^{2} / w_{C_{1}}^{2}\right)$, where $\rho$ is the transverse distance from the cavity axis $z$, and $k_{C_{1,2}}=2 \pi / \lambda_{C_{1,2}}$ [25].

As discussed in connection with Fig. 4 below, for the theoretical curves shown in Figs. 2 and 3, we have chosen only the 30 out of 90 total FORT wells for which $\left|\psi\left(\mathbf{r}_{\mathrm{FORT}}\right)\right| \geq 0.87$, where $\mathbf{r}_{\mathrm{FORT}}$ is such that $U\left(\mathbf{r}_{\mathrm{FORT}}\right)=$ $U_{0}$. Furthermore, for these wells we have averaged $T_{1}\left(\omega_{p}\right)$ over a Gaussian distribution in position $\mathbf{r}$ consistent with a temperature $k_{B} \mathcal{T}=0.1 U_{0}(\sim 200 \mu \mathrm{K})$. Since all parameters are known except for those that characterize atomic motion, the good agreement between theory and experiment [32] allows us to infer that our cooling protocol together with the selection criterion $T_{\text {thresh }}=0.2$ results in individual atoms that are strongly coupled in
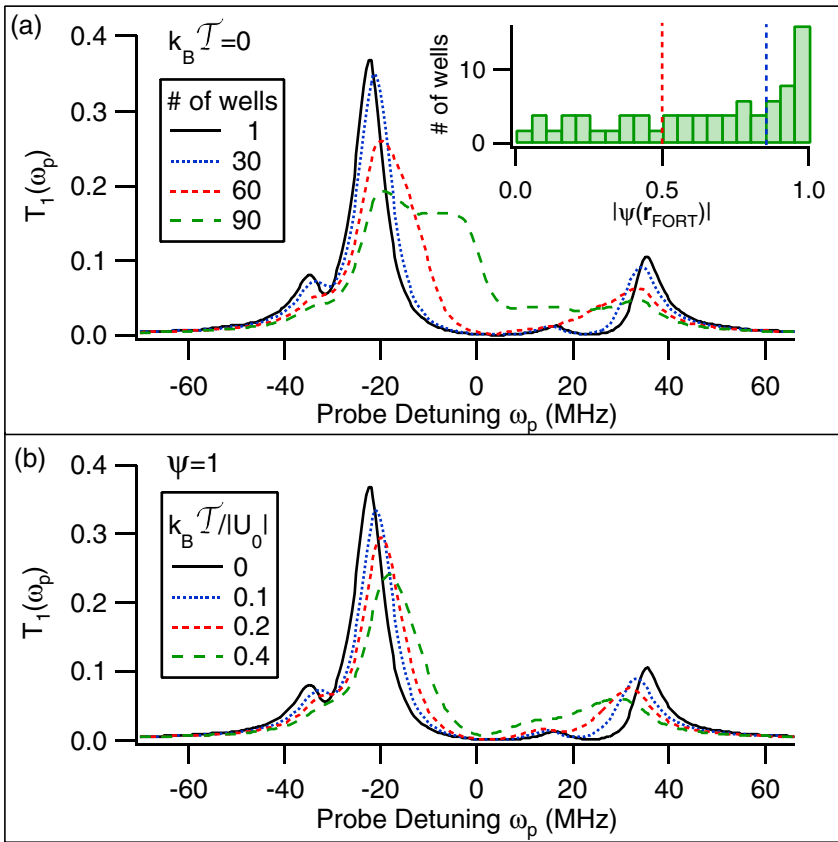

FIG. 4 (color online). Theoretical plots for $T_{1}\left(\omega_{p}\right)$ from the steady-state solution of the master equation. (a) For zero temperature, $T_{1}\left(\omega_{p}\right)$ is calculated from an average over various FORT antinodes along the cavity axis, with the inset showing the associated distribution of values for $\left|\psi\left(\mathbf{r}_{\mathrm{FORT}}\right)\right|$. (b) For an optimum FORT well (i.e., $\left.\left|\psi\left(\mathbf{r}_{\mathrm{FORT}}\right)\right|=1\right), T_{1}\left(\omega_{p}\right)$ is computed for various temperatures from an average over atomic positions within the well. 
one of the "best" FORT wells (i.e., $\left|\psi\left(\mathbf{r}_{\mathrm{FORT}}\right)\right| \geqslant 0.87$ ) with "temperature" $200 \mu \mathrm{K}$ [33].

In support of these assertions, Fig. 4(a) explores the theoretical dependence of $T_{1}\left(\omega_{p}\right)$ on the set of FORT wells selected, and hence on the distribution of values for $\left|\psi\left(\mathbf{r}_{\mathrm{FORT}}\right)\right|$ in the ideal case $\mathcal{T}=0$. Extending the average beyond the 30 "best" FORT wells leads to spectra that are inconsistent with our observations in Figs. 2 and 3. Figure 4(b) likewise investigates the theoretical dependence of $T_{1}\left(\omega_{p}\right)$ on the temperature $\mathcal{T}$ for an atom at an antinode of the FORT with optimal coupling (i.e., $|\psi(\mathbf{r})|=1)$. For temperatures $\mathcal{T} \geqslant 200 \mu \mathrm{K}$, the calculated spectra are at variance with the data in Figs. 2 and 3, from which we infer atomic localization $\Delta z \simeq 33 \mathrm{~nm}$ in the axial direction and $\Delta x=\Delta y \simeq 3.9 \mu \mathrm{m}$ in the plane transverse to the cavity axis. Beyond these conclusions, a consistent feature of our measurements is that reasonable correspondence between theory and experiment is only obtained by restricting $|\psi(\mathbf{r})| \geqslant 0.8$.

Our experiment represents an important advance in the quest to obtain single atoms trapped with optimal strong coupling to a single mode of the electromagnetic field. The vacuum Rabi splitting is the hallmark of strong coupling for single atoms and photons, and all measurements until now have required averaging over many atoms for its observation. By contrast, we are able to observe spectra $T_{1}\left(\omega_{p}\right)$ on an atom-by-atom basis with clearly resolved normal-mode splittings. These spectra contain detailed quantitative information about the coherent coupling $g(\mathbf{r})$ and FORT shifts for each atom. This information indicates that the coupling $g$ is in a narrow range of near-maximal values. Our observations are made possible by the implementation of a new scheme to cool both the radial and axial atomic motion. The capabilities demonstrated in this Letter should provide the tools necessary to implement diverse protocols in quantum information science [5-9].

We gratefully acknowledge the contributions of T. Northup. This research is supported by the Caltech MURI Center for Quantum Networks, by the National Science Foundation, and by the Advanced Research and Development Activity (ARDA).

[1] H. J. Kimble, Phys. Scr., T 76, 127 (1998).

[2] E.T. Jaynes and F.W. Cummings, Proc. IEEE 51, 89 (1963).

[3] J. J. Sanchez-Mondragon, N. B. Narozhny, and J. H. Eberly, Phys. Rev. Lett. 51, 550 (1983).

[4] G. S. Agarwal, Phys. Rev. Lett. 53, 1732 (1984).

[5] T. Pellizzari, S. A. Gardiner, J. I. Cirac, and P. Zoller, Phys. Rev. Lett. 75, 3788 (1995).

[6] L.-M. Duan and H. J. Kimble, Phys. Rev. Lett. 92, 127902 (2004)

[7] J. I. Cirac, P. Zoller, H. J. Kimble, and H. Mabuchi, Phys. Rev. Lett. 78, 3221 (1997).
[8] H.-J. Briegel et al., in The Physics of Quantum Information, edited by D. Bouwmeester, A. Ekert, and A. Zeilinger (Springer, Berlin, 2000), p. 192.

[9] H. Mabuchi and A.C. Doherty, Science 298, 1372 (2002).

[10] Cavity Quantum Electrodynamics, edited by P. Berman (Academic Press, San Diego, 1994).

[11] R. J. Thompson, G. Rempe, and H. J. Kimble, Phys. Rev. Lett. 68, 1132 (1992).

[12] J. J. Childs et al., Phys. Rev. Lett. 77, 2901 (1996).

[13] R. J. Thompson, Q. A. Turchette, O. Carnal, and H. J. Kimble, Phys. Rev. A 57, 3084 (1998).

[14] M. Brune et al., Phys. Rev. Lett. 76, 1800 (1996).

[15] C. J. Hood, M. S. Chapman, T.W. Lynn, and H. J. Kimble, Phys. Rev. Lett. 80, 4157 (1998).

[16] J. Ye, D. W. Vernooy, and H. J. Kimble, Phys. Rev. Lett. 83, 4987 (1999).

[17] J. McKeever et al., Phys. Rev. Lett. 90 , 133602 (2003).

[18] P. Maunz et al., quant-ph/0406136.

[19] M. Riebe et al., Nature (London) 429, 734 (2004).

[20] M. D. Barrett et al., Nature (London) 429, 737 (2004).

[21] A. Wallraff et al., Nature (London) 431, 162 (2004); I. Chiorescu et al., ibid. 431, 159 (2004).

[22] See J. McKeever, Ph.D thesis, California Institute of Technology, 2004 for more technical details.

[23] Birefringence in the mirrors leads to two nondegenerate cavity modes with orthogonal polarizations $\hat{l}_{ \pm}$and mode splitting $\Delta \nu_{C_{1}}=4.4 \pm 0.2 \mathrm{MHz}$. $\mathcal{E}_{\mathrm{FORT}}\left(\mathcal{E}_{\text {Raman }}\right)$ is linearly polarized and aligned close to $\hat{l}_{+}\left(\hat{l}_{-}\right)$for the higher (lower) frequency mode.

[24] K. L. Corwin et al., Phys. Rev. Lett. 83, 1311 (1999).

[25] At the cavity center $z=0$, the mode waists $w_{C_{1,2}}=$ $\{23.4,24.5\} \mu \mathrm{m}$ at $\lambda_{C_{1,2}}=\{852.4,935.6\} \mathrm{nm}$.

[26] $T_{1}\left(\omega_{p}\right)$ is proportional to the ratio of photon flux transmitted by $M_{2}$ to the flux $\left|\mathcal{E}_{p}\right|^{2}$ incident upon $M_{1}$, with normalization $T_{0}\left(\omega_{p}=\omega_{C_{1}}\right) \equiv 1$ for the empty cavity. Figures 2 and 3 also show the corresponding intracavity photon number $\left\langle n\left(\omega_{p}\right)\right\rangle$.

[27] Relative to $\hat{l}_{ \pm}$[23], the linear polarization vector $\hat{l}_{p}$ for the probe field $\mathcal{E}_{p}$ is aligned along a direction $\hat{l}_{p}=$ $\cos \theta \hat{l}_{+}+\sin \theta \hat{l}_{-}$, where $\theta=13^{\circ}$ for Fig. 2; however, the theory is relatively insensitive to $\theta$ for $\theta \leqslant 15^{\circ}$.

[28] The efficiency for photon escape from the cavity is $\alpha_{e 2}=$ $0.6 \pm 0.1$. The propagation efficiency from $M_{2}$ to detectors $\left(D_{1}, D_{2}\right)$ is $\alpha_{P}=0.41 \pm .03$, with then each detector receiving half of the photons. The avalanche photodiodes $\left(D_{1}, D_{2}\right)$ have quantum efficiencies $\alpha_{P}=0.49 \pm 0.05$.

[29] D. Boiron et al., Phys. Rev. A 53, R3734 (1996).

[30] The ac-Stark shifts of the $\left(F^{\prime}=5^{\prime}, m_{F}^{\prime}\right)$ states are given by $\left\{m_{F}^{\prime}, U_{m_{r}^{\prime}}\right\}=\{ \pm 5,1.18 U\},\{ \pm 4,1.06 U\},\{ \pm 3,0.97 U\}$, $\{ \pm 2,0.90 U\},\{ \pm 1,0.86 U\},\{0,0.85 U\}$.

[31] Transmission spectra $T_{1}\left(\omega_{p}\right), \bar{T}_{1}\left(\omega_{p}\right)$ are insensitive over a range of selection criteria $0.02 \leq T_{\text {thresh }} \leq 0.73$.

[32] In Figs. 2 and 3 the discrepancy between experiment and the steady-state theory for $\bar{T}_{1}\left(\omega_{p}\right)$ around $\omega_{p} \sim 0$ can be accounted for by a transient solution to the master equation which includes optical pumping effects over the probe interval $\Delta t_{\text {probe }}$.

[33] Although the spectra are consistent with a thermal distribution, we do not exclude a more complex model involving probe-dependent heating and cooling effects. 